\title{
Noninvasive monitoring of early antiangiogenic therapy response in human nasopharyngeal carcinoma xenograft model using MRI with RGD-conjugated ultrasmall superparamagnetic iron oxide nanoparticles
}

This article was published in the following Dove Press journal:

International Journal of Nanomedicine

14 November 2016

Number of times this article has been viewed

\author{
Yanfen Cuil,* \\ Caiyuan Zhangl,* \\ Ran Luo' \\ Huanhuan Liu' \\ Zhongyang Zhang' \\ Tianyong $\mathrm{Xu}^{2}$ \\ Yong Zhang ${ }^{2}$ \\ Dengbin Wang' \\ 'Department of Radiology, Xinhua \\ Hospital, Shanghai Jiao Tong University \\ School of Medicine, ${ }^{2}$ MR Advanced \\ Application and Research Center, \\ GE Healthcare China, Shanghai, \\ People's Republic of China \\ *These authors contributed equally \\ to this work
}

\begin{abstract}
Purpose: Arginine-glycine-aspartic acid (RGD)-based nanoprobes allow specific imaging of integrin $\alpha v \beta 3$, a protein overexpressed during angiogenesis. Therefore, this study applied a novel RGD-coupled, polyacrylic acid (PAA)-coated ultrasmall superparamagnetic iron oxide (USPIO) (referred to as RGD-PAA-USPIO) in order to detect tumor angiogenesis and assess the early response to antiangiogenic treatment in human nasopharyngeal carcinoma (NPC) xenograft model by magnetic resonance imaging (MRI).
\end{abstract}

Materials and methods: The binding specificity of RGD-PAA-USPIO with human umbilical vein endothelial cells (HUVECs) was confirmed by Prussian blue staining and transmission electron microscopy in vitro. The tumor targeting of RGD-PAA-USPIO was evaluated in the NPC xenograft model. Later, mice bearing NPC underwent MRI at baseline and after 4 and 14 days of consecutive treatment with Endostar or phosphate-buffered saline ( $n=10$ per group).

Results: The specific uptake of the RGD-PAA-USPIO nanoparticles was mainly dependent on the interaction between RGD and integrin $\alpha v \beta 3$ of HUVECs. The tumor targeting of RGD-PAA-USPIO was observed in the NPC xenograft model. Moreover, the T2 relaxation time of mice in the Endostar-treated group decreased significantly compared with those in the control group both on days 4 and 14, consistent with the immunofluorescence results of CD31 and CD61 $(P<0.05)$.

Conclusion: This study demonstrated that the magnetic resonance molecular nanoprobes, RGD-PAA-USPIOs, allow noninvasive in vivo imaging of tumor angiogenesis and assessment of the early response to antiangiogenic treatment in NPC xenograft model, favoring its potential clinical translation.

Keywords: magnetic resonance imaging, ultrasmall superparamagnetic iron oxide, integrin $\alpha v \beta 3$, antiangiogenic therapy, Endostar, nasopharyngeal carcinoma

\section{Introduction}

Tumor angiogenesis, one of the hallmarks of cancer, is a critical factor involved in the tumor growth, invasion, and metastasis and, consequently, is a target for cancer treatment. ${ }^{1,2}$ Nasopharyngeal carcinoma (NPC), one of the most common malignancies in Southern Asia, is known to be highly vascularized. Focus on angiogenesis factors, antiangiogenic agents alone or in conjunction with chemoradiotherapy, has proved to be an effective treatment in advanced NPC., ${ }^{3,4}$ However, not all patients would
Correspondence: Dengbin Wang Department of Radiology, Xinhua Hospital, Shanghai Jiao Tong University School of Medicine, Shanghai, 200092,

People's Republic of China

Tel +86 2l 25077030

Email dbwang8@aliyun.com 
benefit from such treatment with a specific antiangiogenic agent. ${ }^{5,6}$ Therefore, the ability to noninvasively visualize tumor angiogenesis can not only help tailor antiangiogenic treatment by optimizing dosages and timing of drug cycles but also be an elegant approach for monitoring the early therapeutic efficacy. ${ }^{7,8}$

Integrin $\alpha v \beta 3$, a marker of angiogenesis, is responsible for the regulation of endothelial cell activation, migration, proliferation, and differentiation. ${ }^{9}$ It is highly expressed on activated and proliferating endothelial cells during the onset of tumor angiogenesis, rather than quiescent blood vessels, ${ }^{10}$ making it being investigated as a well-characterized molecular imaging marker of tumor-induced angiogenesis. ${ }^{11}$

Peptides containing arginine-glycine-aspartic acid (RGD) can specifically and strongly bind to integrin $\alpha v \beta 3$; therefore, a vast array of RGD-based probes have been developed for early detection of tumor angiogenesis using different modalities, including positron emission tomography, optical imaging, ultrasound imaging, and magnetic resonance imaging (MRI), and some radioactive traces are undergoing clinical trials. ${ }^{12-18}$ Of these modalities, MRI provides the distinct advantage of high spatial resolution and excellent soft tissue contrast without ionizing radiation, as well as the ability to noninvasively detect shallow and deep tumors. However, the inherent insensitivity of MRI hindered their applicability for molecular imaging. In this context, ultrasmall superparamagnetic iron oxide (USPIO) nanoparticles hold a great promise for use as negative magnetic resonance (MR) contrast agents with high relaxivities in terms of their intrinsic properties and versatile surface functionality. ${ }^{19,20}$ Currently, USPIOs conjugated to specific ligands such as RGD peptides to detect tumor are of particular interest. We previously demonstrated that integrin $\alpha v \beta 3$-targeted USPIOs enabled researchers to distinguish tumors with different degrees of integrin $\alpha v \beta 3$ expression. ${ }^{21,22}$ However, few studies have investigated the feasibility of these RGD-based nanoprobes to evaluate the antiangiogenic therapeutic response, especially in the territory of human NPC. ${ }^{23-25}$

Therefore, the purpose of the present study was to validate a novel RGD-coupled, polyacrylic acid (PAA)-coated USPIO (RGD-PAA-USPIO) nanoparticles, for its ability to detect tumor angiogenesis in human NPC xenograft model by MRI and also to investigate its feasibility for monitoring the early response to Endostar antiangiogenic therapy.

\section{Materials and methods}

\section{General materials}

Iron(III) acetylacetonate $\left(\mathrm{Fe}(\mathrm{acac})_{3} \geq 97 \%\right)$ were purchased from Sigma-Aldrich Chemical Co (St Louis, MO,
USA). The RGD peptides, cyclo(Arg-Gly-Asp-Tyr-Lys) (c(RGDyK)), were synthesized by Chinese Peptides Co., Ltd. (Hangzhou, People's Republic of China). The female BALB/c nude mice (6-8 weeks old, 18-22 g body weight) were purchased from Shanghai Experimental Animal Center. The Endostar was provided by Simcere Pharmaceutical Research Co., Ltd.

\section{Synthesis and characterization of RGD- PAA-USPIO nanoparticles}

USPIO was coated by PAA using polyol method. ${ }^{26}$ In brief, a mixture of iron(III) acetylacetonate $(353 \mathrm{mg})$ and PAA (72 mg) was dispersed into a mechanically stirred solution of triethylene glycol $(30 \mathrm{~mL})$. Subsequently, the suspension was heated to $185^{\circ} \mathrm{C}$ for $30 \mathrm{~min}$ under nitrogen gas atmosphere, and then heated to $287^{\circ} \mathrm{C}$ with the heating rate of $10^{\circ} \mathrm{C} / 3 \mathrm{~min}$ and this temperature was maintained for $30 \mathrm{~min}$. The black colloidal suspension was subsequently cooled down to room temperature, retrieved by a magnet, washed three times with ethanol and finally dispersed into deionized water.

For coupling the RGD peptides, $500 \mathrm{mg}$ PAA-USPIO $(2 \mathrm{mg} / \mathrm{mL})$ and $50 \mathrm{mg} \mathrm{c}(\mathrm{RGDyK})$ peptides were dispersed into the solution of 1-ethyl-3-(dimethylaminopropyl)carbodiimide hydrochloride $(5.6 \mathrm{mg})$ dissolved in $100 \mu \mathrm{L}$ of deionized water and thereafter placed into vertical rotating apparatus for $2 \mathrm{~h}$. Subsequently, the mixture was washed with deionized water and centrifuged twice each for $10 \mathrm{~min}$ at 4,000 rpm. Finally, RGD-PAA-USPIO nanoparticles were obtained and dissolved in deionized water. The particle size and distribution characterization of the RGD-PAA-USPIO nanoparticles were measured by transmission electron microscopy (TEM, JEOL-100CX), and the hydrodynamic diameter of them was analyzed by dynamic light scattering (DLS). The relaxivities (in L. $\mathrm{mmol}^{-1} \cdot \mathrm{sec}^{-1}$ ) were determined by a linear fit of the relaxation rate as a function of iron concentration (in mmol/L) using a 1.41-T MRI contrast agent analyzer (mq60; Bruker, Germany) in combination with a custom-made radiofrequency coil.

\section{Cell lines}

Human umbilical vein endothelial cells (HUVECs), purchased from American Type Culture Collection (ATCC, Manassas, VA, USA), were cultured in DMEM culture medium. The CNE-2 cell line (FDCC, Fu Dan IBS Cell Center), a poorly differentiated human NPC cell line, was maintained in RPMI-1640 culture medium. Both the culture media were supplemented with $1 \%$ penicillin-streptomycin and $10 \%$ fetal bovine serum (Gibco, Paisley, UK) at $37^{\circ} \mathrm{C}$ and $5 \% \mathrm{CO}_{2}$ atmosphere. According to a previously study, 
there is no constitutive integrin $\alpha v \beta 3$ expression on the surface of CNE-2 cells. ${ }^{27}$

\section{In vitro evaluation of specificity of the RGD-PAA-USPIO probe}

\section{Prussian blue staining}

HUVECs were diluted to $10^{6}$ cells/mL in DMEM, seeded on glass coverslips in 6-well culture plates, and then co-cultured with $0.03 \mu \mathrm{mol} \mathrm{mL}{ }^{-1}$ PAA-USPIO or RGD-PAA-USPIO for $30 \mathrm{~min}, 1 \mathrm{~h}$, and $3 \mathrm{~h}$, respectively. To further test whether the specificity was induced by integrin $\alpha v \beta 3$, competition experiments were done by adding the free RGD peptides to the RGD-PAA-USPIO in a ratio of 10,000:1. After incubation, the culture medium was removed. The adherent cells were washed three times with phosphate-buffered saline (PBS, $\mathrm{pH}=7.4$ ), fixed by $4 \%$ paraformaldehyde for $30 \mathrm{~min}$. Later, the fixed cells were incubated with the solution of $2 \%$ potassium ferrocyanide and hydrochloric acid ( $\mathrm{V}: \mathrm{V}=1: 1)$ for $10 \mathrm{~min}$, and counterstained with $0.1 \%$ nuclear fast red for $5 \mathrm{~min}$.

\section{Transmission electron microscopy (TEM)}

HUVECs treated with $0.03 \mu \mathrm{mol} \mathrm{mL}^{-1}$ PAA-USPIO or RGD-PAA-USPIO for 30 min were collected, transferred to $1.5 \mathrm{~mL}$ Eppendorf tube, fixed with $2.5 \%$ glutaraldehyde at $4^{\circ} \mathrm{C}$ for $4 \mathrm{~h}$, dehydrated with ethyl alcohol in gradient, and then embedded with mixture of embedding medium and acetone $(\mathrm{V}: \mathrm{V}=1: 1)$ for ultrathin sectioning. Subsequently, the specimen was stained with $2 \%$ uranyl acetate and lead citrate and then observed under TEM at $100 \mathrm{kV}$.

\section{Inductively coupled plasma mass spectrometry (ICP-MS)}

For quantitative assessment of the intracellular iron content, HUVECs incubated with plain USPIO or RGD-PAA-USPIO was digested with $0.25 \%$ trypsogen. A total of $7 \times 10^{5}$ cells were incubated with $37 \%$ hydrochloric acid and heated to $75^{\circ} \mathrm{C}$ for $30 \mathrm{~min}$. Later, ICP-MS (Element 2; Thermo Electron, Bremen, Germany) was used to measure the amount of iron uptake in cells. Iron content was expressed in picogram of iron per cell.

\section{Animal models and treatment}

All the experimental protocols were performed in accordance with the guidelines of Institutional Animal Care and Use Committee of Xinhua Hospital Affiliated to Shanghai Jiao Tong University School of Medicine, and the study was approved by the Animal Ethical and Welfare Committee of Xinhua Hospital. Briefly, female BALB/c nude mice were subcutaneously injected with $0.2 \mathrm{~mL} \mathrm{CNE}-2$ cells $\left(2 \times 10^{6}\right)$ into the right hind flank. Tumors were allowed to grow to $\sim 200 \mathrm{~mm}^{3}$ and then were randomly allocated to either the control group $(n=10)$ or treatment group $(n=10)$. Mice in the treatment group were administered with Endostar intraperitoneally at the dose of 20 $\mathrm{mg} / \mathrm{kg} / \mathrm{d}$ for consecutive 14 days, whereas mice in the control group received PBS at the same dose. Later, all mice underwent MRI at baseline (day 0) and 4 and 14 days after treatment with Endostar or PBS. To evaluate therapeutic response, tumor growth was measured regularly with caliper and calculated using the following formula: $\mathrm{V}=1 / 2 * \mathrm{ab}^{2}$ ( $\mathrm{a}$, length; $\mathrm{b}$, width). The animal's weight was recorded regularly.

\section{In vivo $M R I$ and image analysis}

MRI studies were performed on a clinical 3.0 T MRI system (SignaHDxt; GE Medical System, Milwaukee, WI, USA) equipped with a dedicated 8-channel receiver coil with $2.5 \mathrm{~cm}$ inner diameter (Chenguang Medical Technologies Co., Shanghai, People's Republic of China). All mice were anesthetized with an intraperitoneal injection of pentobarbital sodium (50 mg/kg body weight; Sinopharm, Shanghai, People's Republic of China). For RGD-PAA-USPIO binding evaluation, an additional cohort of mice without any treatment was imaged before and $6 \mathrm{~h}$ after intravenous injection with RGD-PAA-USPIO (5 mice) or PAA-USPIO (5 mice) at the same dose of $300 \mu \mathrm{mol} \mathrm{Fe} / \mathrm{Kg}$. A blocking study with previous injection of $0.30 \mathrm{mM} \mathrm{c}(\mathrm{RGDyK}) 1 \mathrm{~h}$ before intravenous administration of RGD-PAA-USPIO (5 mice) was also conducted. For treatment evaluation, all mice in the control and Endostar-treated group were administered RGD-PAA-USPIO before therapy and 4 and 14 days after treatment initiation to determine the therapeutic effects on tumor vasculature. The following sequences were adopted in data acquisition: 1) Transverse T2-weighted fast spin echo imaging for morphology: TR/TE, 3,000 ms/102.4 ms; field of view (FOV), $60 \times 60 \mathrm{~mm}^{2}$; matrix, $64 \times 64$; slice thickness/ interval, $1.2 \mathrm{~mm} / 0 \mathrm{~mm}$. 2) T2 mapping for the calculation of T2 and R2 values: TR, 1,000 ms; TE $=8.25-66.0 \mathrm{~ms}$ ( 8 echoes, $8.25 \mathrm{~ms}$ interval between two echoes); FOV, $60 \times 60 \mathrm{~mm}^{2}$; matrix, $192 \times 128$; slice thickness/interval, $1.2 \mathrm{~mm} / 0 \mathrm{~mm}$; number of excitations $(\mathrm{NEX})=2$. For each data set, regions of interest were manually drawn on the center slice of the tumor after identification of the solid part of the tumor on conventional T2WI by one radiologist (CZ, with 9 years of experience in MRI analysis) who was blinded to the experimental design. Later, T2 and R2 values of the tumors were automatically calculated by the T2 Map and R2 Star program on the dedicated post-processing workstation (ADW4.3, GE Healthcare). $\Delta \mathrm{T} 2$ are calculated by subtracting the post-contrast value of $\mathrm{T} 2$ from its pre-contrast value. 


\section{Histological study}

After MRI, tumor tissues were surgically removed from the sacrificed mice and were then embedded, frozen in liquid nitrogen, and cut into $5 \mu \mathrm{m}$ frozen slices. Frozen thin sections were fixed in acetone and were air dried. After blocking with $2 \%$ BSA, slices were incubated with rat anti-mouse CD31 antibody (1:100; BD Biosciences, San Jose, CA, USA) and hamster anti-mouse CD61 (integrin $\beta 3$ ) antibody (1:200; BD Biosciences) and then visualized with FITC-conjugated goat anti-rat and $\mathrm{Cy} 3$-conjugated goat anti-hamster secondary antibody (1:200; Santa Cruz Biotechnology, CA, USA). Subsequently, nuclei were stained with 4'-6-diamidino-2phenylindole. Fluorescence images were acquired with an epifluorescence microscope (Olympus, X81).

All quantitative analyses of the positive area of CD61 and CD31-positive vessels on the immunofluorescence staining were assessed by using ImageJ software. The pathologist who performed the immunohistochemistry analysis was blinded to the experimental groups. For each tumor, at least 4 randomly selected vision fields from the representative tumor slices were analyzed.

To directly visualize RGD-PAA-USPIO targeting the angiogenic vessels, Prussian blue staining of tumor tissues in the additional group of binding evaluation was performed, and the procedure was the same as that described in the section "In vitro evaluation of specificity of the RGD-PAAUSPIO probe."

\section{Statistical analysis}

All quantitative data were expressed as mean \pm standard deviation. Statistical analysis was performed by using the Student's $t$-test or Mann-Whitney $U$-test with GraphPad Prism 5.0 (GraphPad Software Inc., La Jolla, CA). $P$-value $<0.05$ was considered statistically significant.

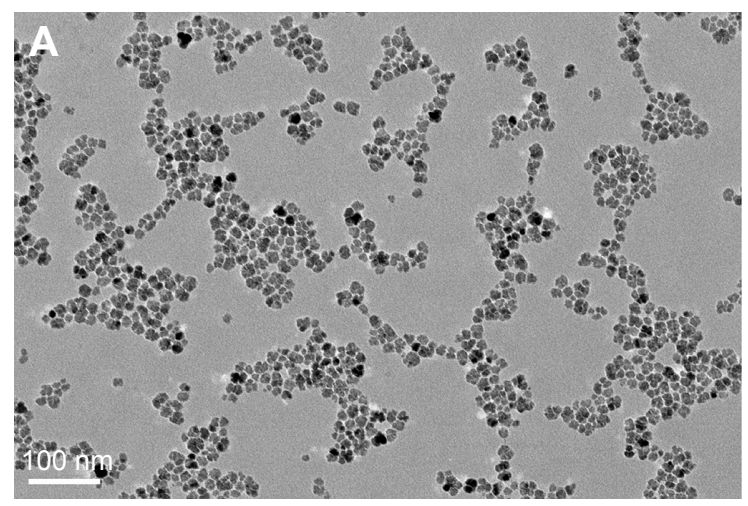

Figure I Characterization of RGD-PAA-USPIO probes.

Note: (A and B) Representative TEM images of RGD-PAA-USPIO nanoparticles.

Abbreviations: PAA, polyacrylic acid; RGD, arginine-glycine-aspartic acid; TEM, transmission electron microscopy; USPIO, ultrasmall superparamagnetic iron oxide.

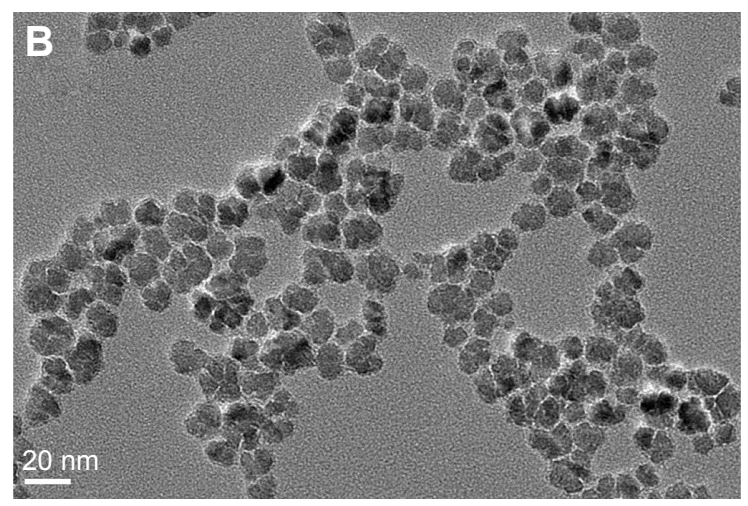

\section{Results \\ Characterization of RGD-PAA-USPIO probes}

The core size of the RGD-PAA-USPIO particles determined by TEM was $14.5 \pm 0.1 \mathrm{~nm}$ (Figure 1), and the mean hydrodynamic diameter of these nanoparticles, measured by DLS analysis, was $\sim 59.44 \mathrm{~nm}$. $\mathrm{T}_{1}$ and $\mathrm{T}_{2}$ relaxivity values of RGDPAA-USPIO were measured to be 8.7 and $137.85 \mathrm{mMS}^{-1}$, respectively. Such nanoparticles can be dispersed in water for several months without precipitation, indicating good stability.

\section{Specificity evaluation of the RGD-PAA- USPIO probe in vitro}

The specific binding of the RGD-PAA-USPIO probes for integrin $\alpha v \beta 3$ was assessed by Prussian blue staining (Figure 2 ). After incubation with HUVECs for 30 min, small amounts of iron particles were observed in the RGD-PAA-USPIO group, whereas there was no significant uptake during the plain USPIO particles and the RGD-PAA-USPIO plus free RGD treatments. With prolonged incubation time, the uptake of nanoparticles were obviously increased in all three groups, showing a time-dependent manner. However, competition with free RGD peptide reduced the amount of blue granules in the cytoplasm of HUVECs dramatically.

To further quantify the uptake of nanoparticles, the ICP-MS study was performed. As shown in Figure 3A, the mean cellular iron content in HUVECs incubated with plain USPIO was $0.23 \pm 0.02 \mathrm{pg} / \mathrm{cell}$ after incubating for $30 \mathrm{~min}$, whereas it increased to $0.43 \pm 0.02 \mathrm{pg} /$ cell for cells incubated with RGD-PAA-USPIO. After blocking the integrin $\alpha v \beta 3$ receptor by adding the free ligands, the mean cellular iron content of RGD-PAA-USPIO incubated cells decreased to $0.37 \pm 0.02 \mathrm{pg} / \mathrm{cell}$. In line with the Prussian blue findings, the 




Figure 2 Cellular uptake of particles in HUVECs evaluated by Prussian blue staining.

Notes: The plain USPIO (A-C), RGD-PAA-USPIO (D-F), and RGD-PAA-USPIO + RGD (competition, G-I) were incubated with HUVECs at an iron concentration of $0.03 \mu \mathrm{mol} \mathrm{mL}^{-1}$ for different time intervals $(30 \mathrm{~min}, \mathrm{I} \mathrm{h}$, and $3 \mathrm{~h}$ ). Scale bar: $10 \mu \mathrm{m}$.

Abbreviations: HUVEC, human umbilical vein endothelial cell; PAA, polyacrylic acid; RGD, arginine-glycine-aspartic acid; USPIO, ultrasmall superparamagnetic iron oxide.

mean cellular iron content in HUVECs in all the three groups also showed a time-dependent manner. All these qualitative and quantitative analyses indicated that the accumulation of these nanoparticles was specifically mediated by the interaction between the RGD peptides and integrin $\alpha v \beta 3$.

To identify the subcellular localization of RGD-PAAUSPIO, TEM observations of HUVECs were conducted. As shown in Figure 3B and C, large amounts of RGD-PAAUSPIO nanoparticles were internalized and accumulated in the cellular endosomes. By contrast, the plain USPIOs were mainly deposited outside the HUVECs.

\section{Evaluation of integrin $\alpha v \beta 3$ binding in vivo}

On the basis of in vitro experimental results, the capability of the RGD-PAA-USPIO nanoparticles to bind integrin $\alpha v \beta 3$ in vivo was evaluated in the CNE-2 NPC xenograft model (Figure 4A). For mice receiving RGD-PAA-USPIO, a dramatic MR signal drop was found in the periphery and some central areas of the tumor. However, after competition with preinjection of free RGD peptides, the signal drop was reduced. For mice receiving plain USPIO, the signal intensity was less pronounced and just marginally decreased. Accordingly, quantitative analysis of the decrease of $\mathrm{T} 2$ relaxation time of the tumors was $36.1 \pm 10.8,20.9 \pm 8.5$, and $9.8 \pm 4.3 \mathrm{~ms}$ for mice receiving RGD-PAA-USPIO, RGD-PAA-USPIO plus free RGD peptide, and plain USPIOs, respectively (Figure 4B).

To confirm the RGD-PAA-USPIO targeting specificity histologically, the expression level of integrin $\alpha v \beta 3$ on NPC tumor sections was investigated. On the one hand, there is no integrin $\alpha v \beta 3$ expression on CNE- 2 cell. ${ }^{27}$ On the other hand, the tumor vasculature endothelial cells are of mouse origin and the CNE-2 tumor cells are of human origin in NPC xenograft model. ${ }^{28}$ Furthermore, most of $\beta 3$ was found to bind to $\alpha v(\alpha v \beta 3)$ or $\alpha \operatorname{IIb}(\alpha \operatorname{IIb} \beta 3)$, but in tumors the latter is hardly expressed. Hence, only immunofluorescence with anti-murine integrin $\beta 3$ antibody was performed to confirm the integrin $\alpha v \beta 3$ expression in CNE-2 tumor tissue. As shown in Figure 5, the perfect co-localization of anti-murine integrin $\beta 3$ and $C D 31$ was observed, indicating that integrin $\alpha v \beta 3$ expression in the CNE-2 tumor tissues was mainly derived from the tumor blood vessels. Additionally, Prussian blue staining of tumor tissues identified that large amount of RGD-PAA-USPIO nanoparticles were mainly attached to the interior of the blood vessel surface and competition with free RGD peptide reduced the targeting efficiency, while little plain USPIO particles were mainly distributed in the interstitial space around the tumor 

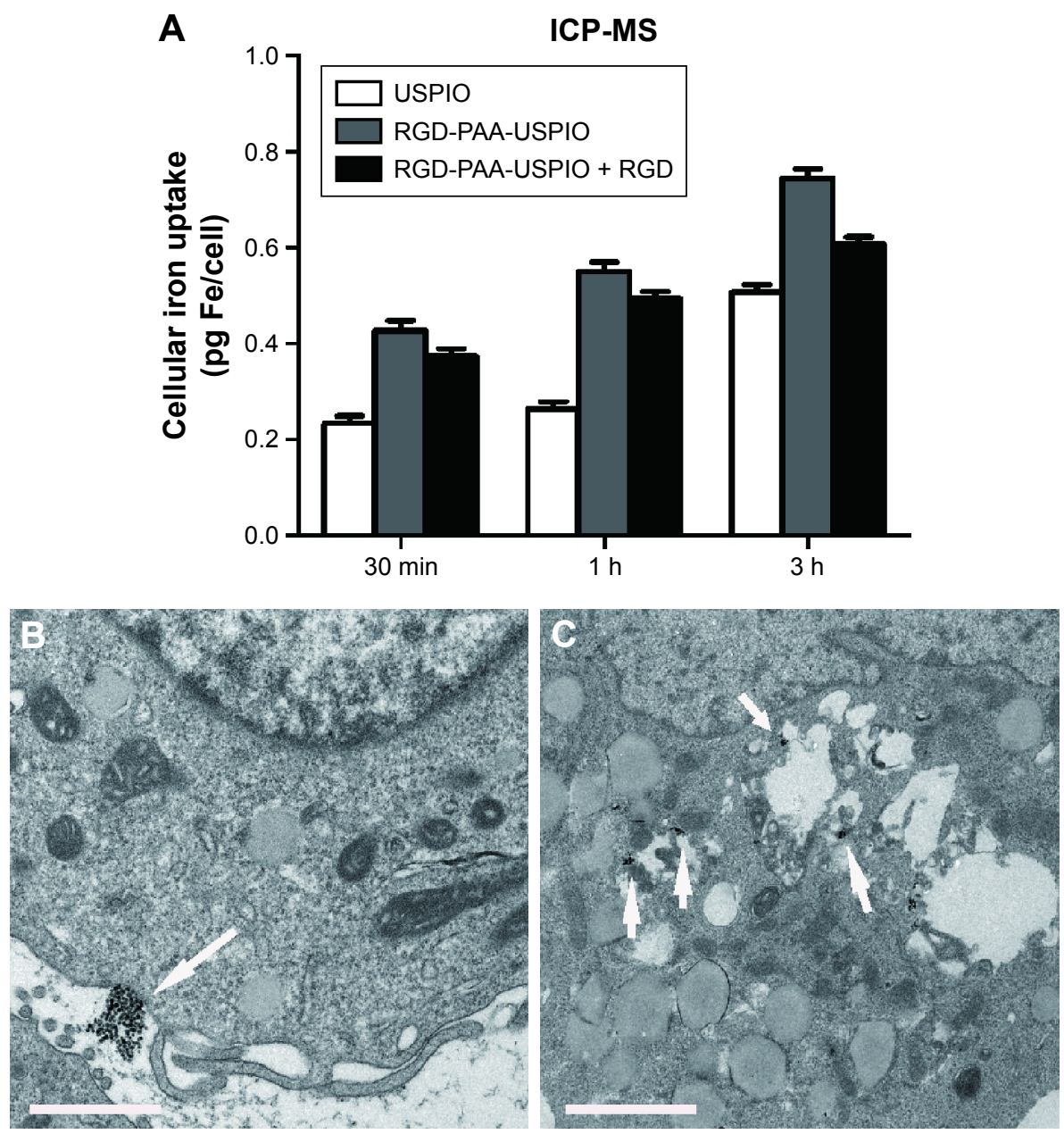

Figure 3 Cellular uptake of particles in HUVECs evaluated by ICP-MS (A) and TEM (B and $\mathbf{C})$.

Notes: Results from ICP-MS measurement of the mean intracellular iron contents after incubation of HUVECs with plain USPIO, RGD-PAA-USPIO, and RGD-PAA-USPIO competed with free RGD (competition) for different time intervals (A). Results from TEM images of the subcellular location in HUVECs after incubation with plain USPIO (B) and RGD-PAA-USPIO (C) for $30 \mathrm{~min}$. Scale bar: I $\mu \mathrm{m}$. The arrows in the figures refer to the iron particle of plain USPIO(B) and RGD-PAA-USPIO(C).

Abbreviations: HUVEC, human umbilical vein endothelial cell; ICP-MS, inductively coupled plasma mass spectrometry; PAA, polyacrylic acid; RGD, arginine-glycine-aspartic acid; TEM, transmission electron microscopy; USPIO, ultrasmall superparamagnetic iron oxide.

vessel (Figure 6). All these results indicate that the RGDPAA-USPIO nanoparticles homing was indeed specifically mediated by RGD-integrin interaction.

\section{Effects of Endostar on CNE-2 tumor growth}

As displayed in Figure 7, a much less time-dependent increase in tumor growth was observed in the treated group than the control, and a significant difference in tumor volume was observed between the two groups from day 8 after therapy onset $(P<0.05)$. No observable side effects were observed during the treatment process.

\section{Monitoring the antiangiogenic effect of Endostar by MRI}

To noninvasively monitor the antiangiogenic therapy response, MRI data sets from all mice in both control and treated groups were analyzed. As shown in Figure 8A, mice in the control group after injection of RGD-PAA-USPIO showed obviously decreased signal intensity in the peripheral areas, whereas mice in the Endostar-treated group showed little signal changes. Quantitative analysis found that both the decrease of T2 relaxation time and post-contrast $\mathrm{R} 2$ relaxation rate of tumors in the control group were much higher than those in the treated group at day 4 and day $14(P<0.05)$ (Figure $8 \mathrm{~B}$ and $\mathrm{C}$ ).

\section{Histological assessment of tumor response}

To confirm the inhibition effect of Endostar histologically, expressions of both integrin $\beta 3$ and CD31 of the tumors were investigated. As shown in Figure 9, a significant higher CD31-positive area fraction in the control group was observed compared to those in the treated group at the end of the treatment $(P<0.05)$. Similar trend was found for the integrin $\beta 3$ 



Figure 4 Evaluation of integrin $\alpha v \beta 3$ binding in vivo.

Notes: (A) T2-weighted MR imaging of tumor before and $6 \mathrm{~h}$ after intravenous injection of RGD-PAA-USPIO, plain USPIO, or RGD-PAA-USPIO plus free RGD peptide. (B) The corresponding T2 relaxation time decrease of tumors before and after probe injection. $* * P<0.01$. The arrow in Figure $4 \mathrm{~A}$ refer to the signal intensity decrease caused by the injection of RGD-PAA-USPIO.

Abbreviations: MR, magnetic resonance; PAA, polyacrylic acid; RGD, arginine-glycine-aspartic acid; USPIO, ultrasmall superparamagnetic iron oxide.

expression $(P<0.05)$. It was also observed that integrin $\beta 3$ predominantly co-localized with the distribution of tumor blood vessels. All these results demonstrated that RGD-PAA-USPIO can be used for noninvasive monitoring of the antiangiogenic therapeutic response in human NPC xenograft model.

\section{Discussion}

Noninvasive visualization and quantification of integrin $\alpha v \beta 3$ expression levels in vivo have greatly assisted the understanding of the mechanisms of angiogenesis and hold a great promise for earlier screening and monitoring the efficacy of 

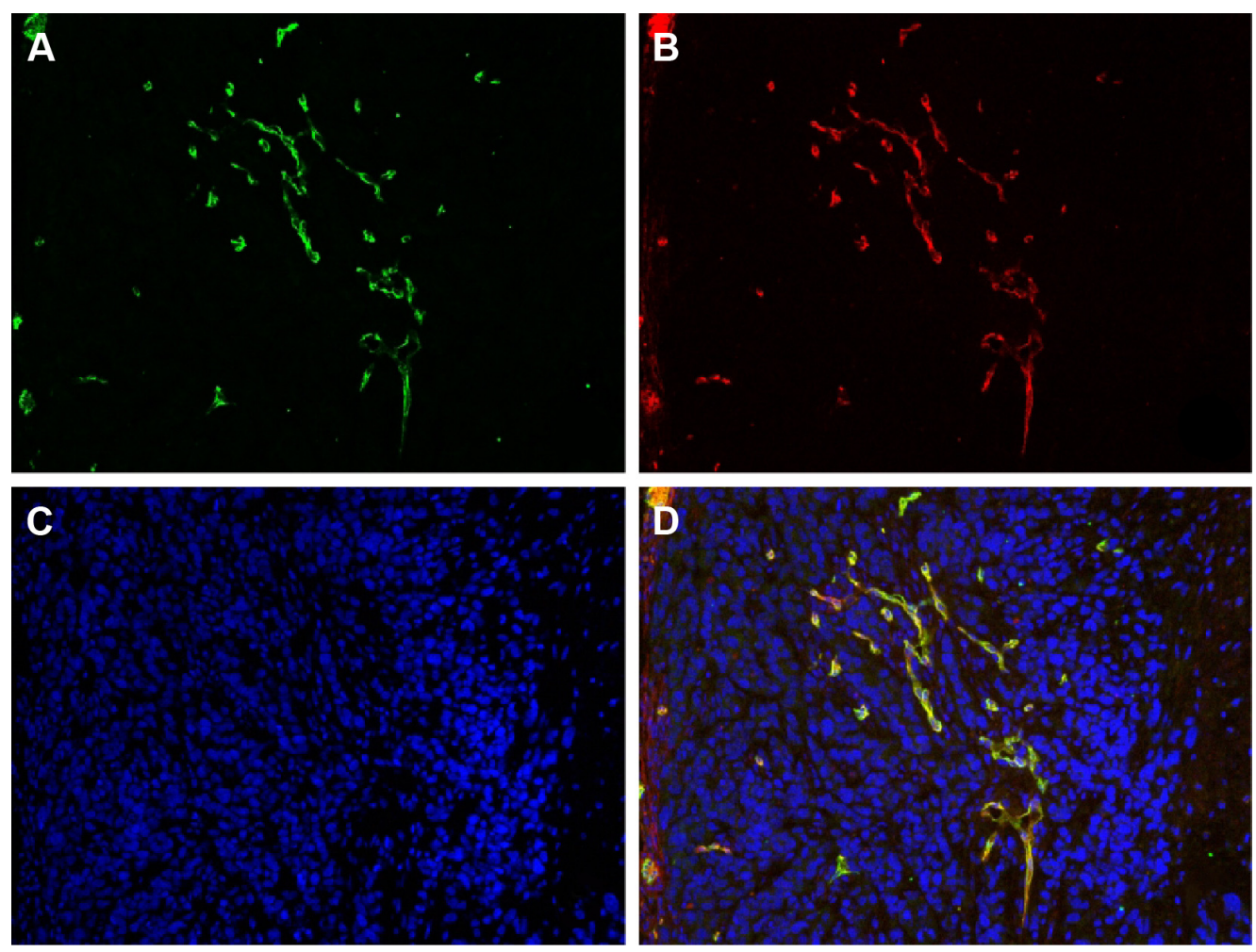

Figure 5 Ex vivo CNE-2 tumor tissue immunofluorescence staining of co-staining (D) of CD3I (A, green) and CD6I (B, integrin $\beta 3$, red), and DAPI (C). Note: Magnification $\times 200$.

Abbreviation: DAPI, 4'-6-diamidino-2-phenylindole.
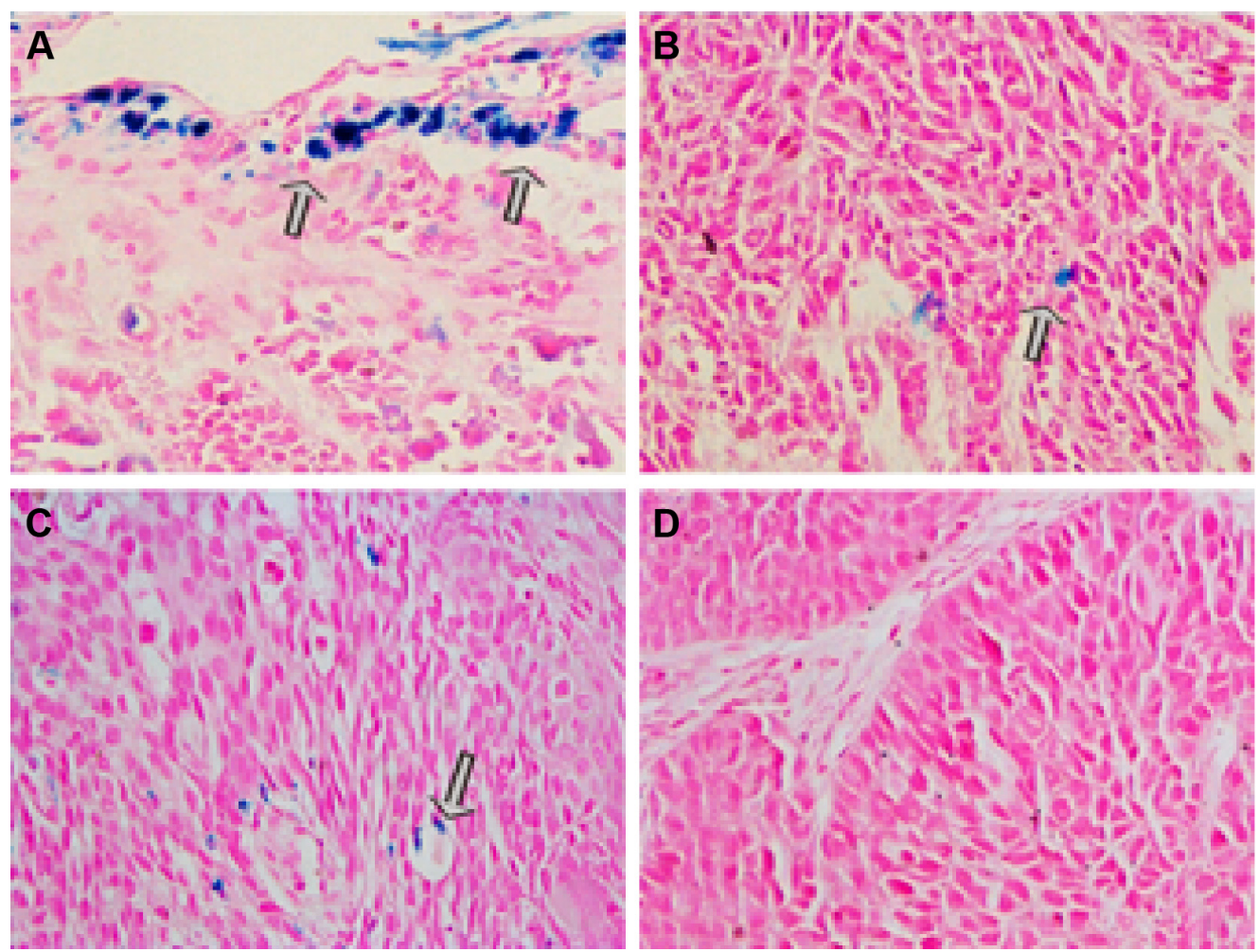

Figure 6 Prussian blue staining of tumor tissues after intravenous injection of RGD-PAA-USPIO (A), plain USPIO (B), or RGD-PAA-USPIO plus free RGD peptides (C) and conventional HE staining (D) $(\times 200)$.

Note: The arrow in the figures refer to the different iron particles of Prussian blue staining from RGD-PAA-USPIO(A), plain USPIO (B), and RGD-PAA-USPIO plus free RGD (C). Abbreviations: HE, hematoxylin and eosin; PAA, polyacrylic acid; RGD, arginine-glycine-aspartic acid; USPIO, ultrasmall superparamagnetic iron oxide. 


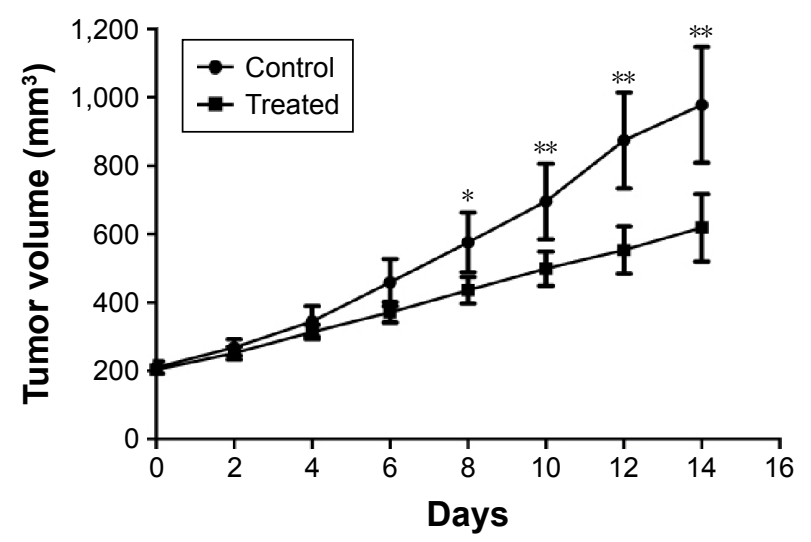

Figure 7 Endostar treatment inhibits growth of CNE-2 tumors.

Notes: Comparison of tumor volumes in the control and Endostar-treated group in CNE-2 xenograft model. Tumor volume was determined by caliper measurements. Error bars denote standard errors. $(* P<0.05 ; * * P<0.01)$. antiangiogenic treatment. This study has demonstrated that MRI with a novel RGD-coupled, PAA-coated USPIO could efficiently identify tumor angiogenesis in the CNE-2 NPC xenograft model. Furthermore, the molecular MRI is capable of noninvasive monitoring of the early treatment response to Endostar antiangiogenic therapy.

This study developed a novel RGD-based MR nanoprobe, implementing PAA coating in terms of hydrophilic nature and the high density of functional carboxylic acid groups, which are compatible with many specific ligands through multivalency effects. Moreover, the cyclic RGD peptide is commercially available and its stability is 30 -fold higher than linear peptide. ${ }^{29}$ In addition, these RGD-PAA-USPIO nanoparticles have well-controlled size, high negative relaxivities,
A

T2WI
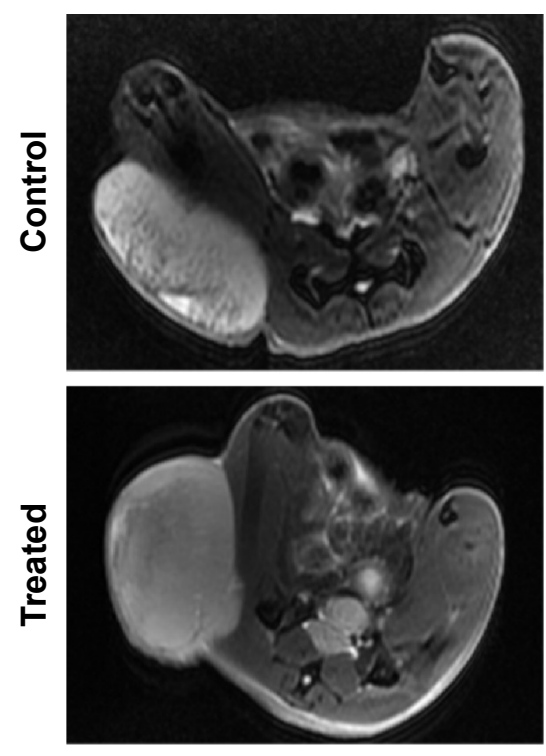

B



T2 mapping
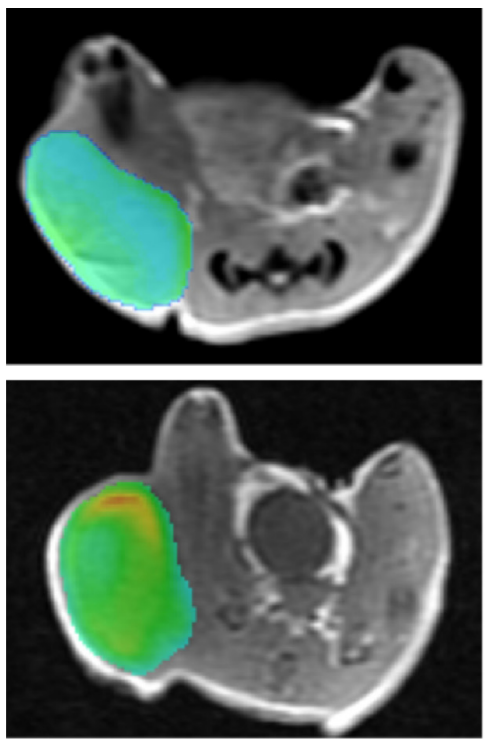

R2 mapping
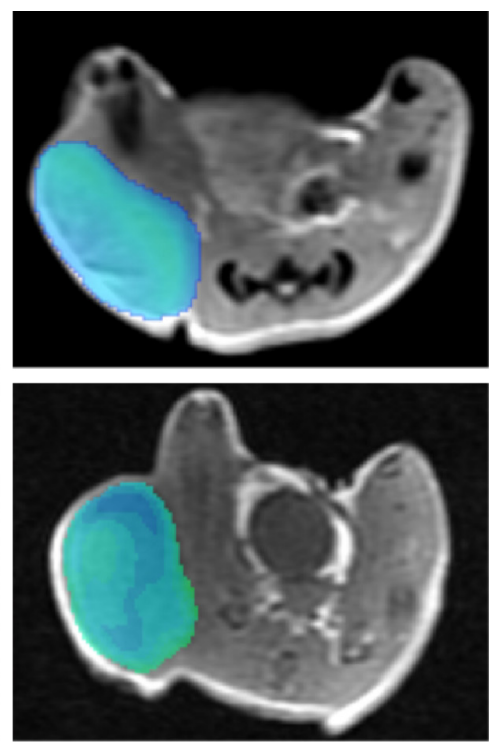

C

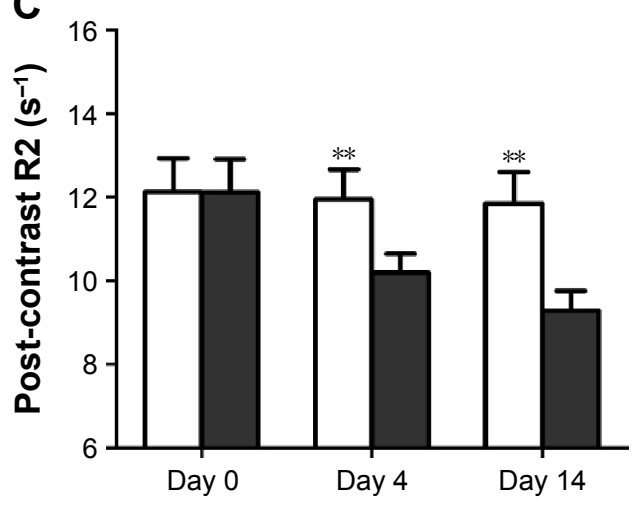

High



Low

Figure 8 Monitoring the antiangiogenic effect of Endostar by MRI.

Notes: (A) Representative T2WI, T2, and R2 mapping images of mice in the control and treatment groups after injection of RGD-PAA-USPIO. (B and C) Quantitative analysis of the decrease of $\mathrm{T} 2$ relaxation time and post-contrast $\mathrm{R} 2$ values of tumors between control and treatment group. $* * P<0.01$.

Abbreviations: MRI, magnetic resonance imaging; PAA, polyacrylic acid; RGD, arginine-glycine-aspartic acid; USPIO, ultrasmall superparamagnetic iron oxide. 

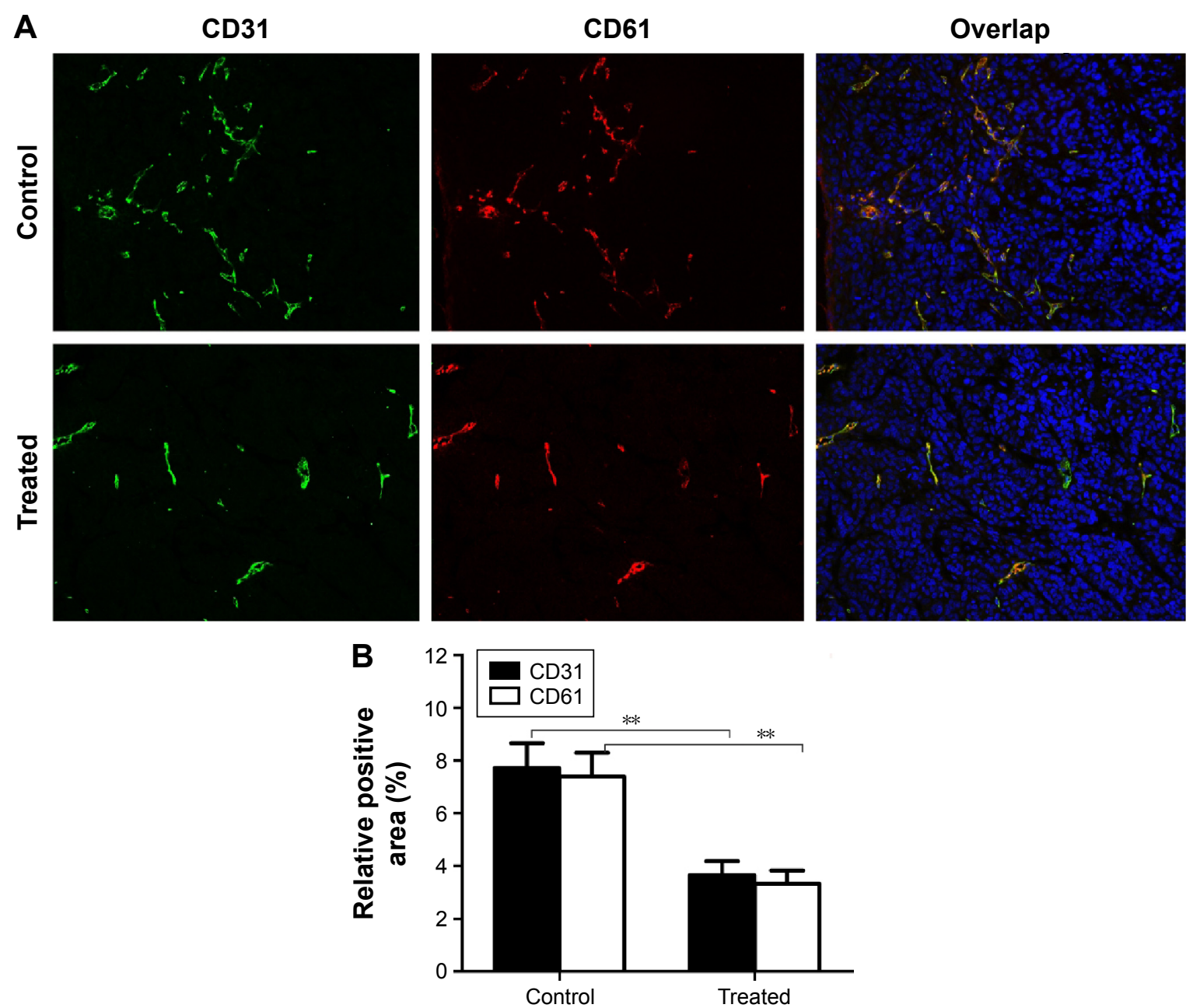

Figure 9 Histological study of tumor tissues.

Notes: (A) Immunofluorescence staining of tumor vasculature and murine integrin $\beta 3$ expression with antibodies against CD3I (green) and CD6I (integrin $\beta 3$, red) in the Endostar-treated and control groups at the end of the study. (B) Quantitative analysis of integrin $\beta 3$ and CD3I-positive area fraction of tumors between control and treatment group. $* * p<0.01$.

and the preparation process is simple and low energy consuming, making it an attractive targeted contrast agent for MRI and potentially suited for clinical translation.

The specificity of the integrin $\alpha v \beta 3$-targeted nanoparticles was investigated in vitro and in vivo. Compared to plain USPIOs, HUVECs had a higher uptake of RGD-PAA-USPIO and could be reduced after inhibition with free RGD peptides, which verified the specific binding of RGD-PAA-USPIO for integrin $\alpha v \beta 3$. Moreover, it also had higher uptake for both internalization through the cell membrane and accumulation within endosome, in line with some previous reports. ${ }^{21,22}$ For in vivo experiment, CNE-2 tumor models were used in which integrin $\alpha v \beta 3$ was solely expressed on the tumor vasculature, similar to most kinds of HNSCC cell line, ${ }^{13,30}$ making these models ideal to investigate the potential of RGD-based probes to image tumor angiogenesis. The results of the present study demonstrated that RGD-PAA-USPIO caused a heterogeneous signal decrease mainly in the periphery and some center areas of the tumor, which is consistent with the characteristics of abundant angiogenesis and integrin $\alpha v \beta 3$ expression pattern of the tumor. In contrast, there was only a low degree of T2 signal reduction for mice with plain USPIO, probably due to extravasation of particles from intratumoral vessels and dispersion into the interstitial space. Quantitative analysis also found that the $\mathrm{T} 2$ relaxation time decreased significantly more after injection of RGD-PAA-USPIO than after injection of plain particles $(P<0.05)$. As proved by Prussian blue staining of tumor tissues, a large amount of RGD-PAA-USPIO nanoparticles mainly targeted tumor angiogenic vessels, while some plain USPIO is found to extravasate into the interstitial space. Taken together, these findings suggest that RGD-PAA-USPIO is able to specifically and efficiently probe the tumor angiogenesis in human NPC xenograft model by using MRI. 
In the past decade, targeting integrin $\alpha v \beta 3$ with RGDbased MR molecular probes has already been investigated for imaging the tumor angiogenesis. ${ }^{17,21,22,31}$ However, MR studies using the RGD-based molecular probes to monitor treatment efficacy are limited, and most of them are paramagnetic gadolinium compounds. ${ }^{7,24,25}$ Therefore, the aforementioned molecular nanoparticles, RGD-PAA-USPIO, were applied to evaluate the early response to Endostar antiangiogenic therapy. Endostar, a novel modified recombinant human endostatin, has showed strong antiangiogenic effect on various xenograft tumor model and cancer patients, including NPC..$^{32-34}$ The results of this study demonstrated that Endostar impeded tumor growth without evident tumor regression and that a significant tumor growth inhibition was not observed until day 8, because Endostar was principally cytostatic rather than cytotoxic. It was also shown that Endostar could reduce the tumor vessels, resulting in the decrease of microvessel density, and subsequently lowered the integrin $\alpha v \beta 3$ expression. To better monitor antiangiogenic therapy response by molecular MRI over common anatomical approaches, a time point of day 4 after treatment was chosen to study the early therapeutic response of Endostar. As shown in Figure 7, a significant difference of the T2 signal decrease was observed at day 4 after the initiation of Endostar treatment between the control and treated groups. Moreover, after a long-term treatment of 14 days, similar results were obtained for the MR data and the relevant histopathological changes in MVD scores as well as integrin $\alpha v \beta 3$ level. Thus, it is reasonable to believe that, besides the diagnostic purpose, RGD-PAA-USPIO may also be used in the assessment of the tumoral early response to antiangiogenic therapy and be potentially used to screen the candidates for such an antiangiogenesis therapy.

However, as for USPIO-based nanoparticles, the nonspecific uptake of the molecular probes by reticuloendothelial system may be high. Therefore, the improvement of the pharmacokinetic profiles by surface modification and size control remains to be the ongoing research. On the other hand, a three-dimensional measurement might be needed for further investigation to provide entire information about the morphologic distribution of tumor angiogenesis indicating the heterogeneity.

\section{Conclusion}

This study demonstrated that MRI with RGD-coupled, PAAcoated USPIOs allowed noninvasive in vivo assessment of tumor angiogenesis by targeting integrin $\alpha v \beta 3$ in human NPC xenograft model. Furthermore, MRI with these molecular nanoprobes was capable of noninvasive monitoring of the early response to antiangiogenic therapeutic efficacy, making it more promising for potentially clinical translation.

\section{Acknowledgments}

The authors thank Chunfu Zhang (Med-X Research Institute, Shanghai Jiao Tong University, People's Republic of China) for technical assistance in the synthesis of nanoparticles. This study was supported by grants from Key Program of Basic Research from Shanghai Municipal Science and Technology Commission (12JC1406500), the National Natural Science Foundation of China (NSFC 81171389, 81371621), the Program of Shanghai Municipal Health Outstanding Discipline Leader (XBR 2013110), and the Program of Medical Engineering Cross Research Fund of Shanghai Jiao Tong University (YG2012MS15). The funders had no role in study design, data collection and analysis, decision to publish, or preparation of the manuscript.

\section{Author contributions}

DW and YC conceived and designed the experiments. YC, $\mathrm{CZ}$, and HL performed the experiments. YC, RL, ZZ, and TX analyzed the data. $\mathrm{CZ}$ designed and synthetized the novel contrast agent. YC and DW prepared the first draft of the manuscript. All authors contributed toward data analysis, drafting and critically revising the paper and agree to be accountable for all aspects of the work.

\section{Disclosure}

The authors report no conflicts of interest in this work.

\section{References}

1. Hanahan D, Weinberg RA. Hallmarks of cancer: the next generation. Cell. 2011;144(5):646-674.

2. Kerbel RS. Antiangiogenic therapy: a universal chemosensitization strategy for cancer? Science. 2006;312(5777):1171-1175.

3. Cao S, Durrani FA, Toth K, Rustum YM, Seshadri M. Bevacizumab enhances the therapeutic efficacy of Irinotecan against human head and neck squamous cell carcinoma xenografts. Oral oncol. 2011;47(6): 459-466.

4. Yoo DS, Kirkpatrick JP, Craciunescu O, et al. Prospective trial of synchronous bevacizumab, erlotinib, and concurrent chemoradiation in locally advanced head and neck cancer. Clin Cancer Res. 2012;18(5): 1404-1414.

5. Sennino B, McDonald DM. Controlling escape from angiogenesis inhibitors. Nat Rev Cancer. 2012;12(10):699-709.

6. Bergers G, Hanahan D. Modes of resistance to anti-angiogenic therapy. Nat Rev Cancer. 2008;8(8):592-603.

7. Mulder WJ, van der Schaft DW, Hautvast PA, et al. Early in vivo assessment of angiostatic therapy efficacy by molecular MRI. FASEB J. 2007;21(2):378-383.

8. Browder T, Butterfield CE, Kraling BM, et al. Antiangiogenic scheduling of chemotherapy improves efficacy against experimental drug-resistant cancer. Cancer Res. 2000;60(7):1878-1886. 
9. Hood JD, Cheresh DA. Role of integrins in cell invasion and migration. Nat Rev Cancer. 2002;2(2):91-100.

10. Brooks PC, Clark RA, Cheresh DA. Requirement of vascular integrin alpha v beta 3 for angiogenesis. Science. 1994;264(5158):569-571.

11. Backer MV, Backer JM. Imaging key biomarkers of tumor angiogenesis. Theranostics. 2012;2(5):502-515.

12. Ellegala DB, Leong-Poi H, Carpenter JE, et al. Imaging tumor angiogenesis with contrast ultrasound and microbubbles targeted to alpha(v) beta3. Circulation. 2003;108(3):336-341.

13. Beer AJ, Grosu AL, Carlsen J, et al. [18F]galacto-RGD positron emission tomography for imaging of alphavbeta3 expression on the neovasculature in patients with squamous cell carcinoma of the head and neck. Clin Cancer Res. 2007;13(22 Pt 1):6610-6616.

14. Park JA, Lee JJ, Jung JC, et al. Gd-DOTA conjugate of RGD as a potential tumor-targeting MRI contrast agent. Chembiochem. 2008;9(17): 2811-2813.

15. Deshpande N, Ren Y, Foygel K, Rosenberg J, Willmann JK. Tumor angiogenic marker expression levels during tumor growth: longitudinal assessment with molecularly targeted microbubbles and US imaging. Radiology. 2011;258(3):804-811.

16. Liu Y, Yang Y, Zhang C. A concise review of magnetic resonance molecular imaging of tumor angiogenesis by targeting integrin alphavbeta3 with magnetic probes. Int J Nanomedicine. 2013;8:1083-1093.

17. Debergh I, Van Damme N, De Naeyer D, et al. Molecular imaging of tumor-associated angiogenesis using a novel magnetic resonance imaging contrast agent targeting alphavbeta 3 integrin. Ann Surg Oncol. 2014;21(6):2097-2104.

18. Liu Z, Liu S, Niu G, Wang F, Liu S, Chen X. Optical imaging of integrin alphavbeta3 expression with near-infrared fluorescent RGD dimer with tetra(ethylene glycol) linkers. Mol Imaging. 2010;9(1):21-29.

19. Huang J, Zhong X, Wang L, Yang L, Mao H. Improving the magnetic resonance imaging contrast and detection methods with engineered magnetic nanoparticles. Theranostics. 2012;2(1):86-102.

20. Huang X, Zhuang J, Chen D, et al. General strategy for designing functionalized magnetic microspheres for different bioapplications. Langmuir. 2009;25(19):11657-11663.

21. Zhang C, Jugold M, Woenne EC, et al. Specific targeting of tumor angiogenesis by RGD-conjugated ultrasmall superparamagnetic iron oxide particles using a clinical 1.5-T magnetic resonance scanner. Cancer Res. 2007;67(4):1555-1562.

22. Jiang $T$, Zhang $C$, Zheng $X$, et al. Noninvasively characterizing the different alphavbeta3 expression patterns in lung cancers with RGDUSPIO using a clinical 3.0T MR scanner. Int J Nanomedicine. 2009;4: 241-249.
23. Zhang F, Huang X, Zhu L, et al. Noninvasive monitoring of orthotopic glioblastoma therapy response using RGD-conjugated iron oxide nanoparticles. Biomaterials. 2012;33(21):5414-5522.

24. Chen WT, Shih TT, Chen RC, Tu SY, Hsieh WY, Yang PC. Integrin alphavbeta3-targeted dynamic contrast-enhanced magnetic resonance imaging using a gadolinium-loaded polyethylene gycol-dendrimercyclic RGD conjugate to evaluate tumor angiogenesis and to assess early antiangiogenic treatment response in a mouse xenograft tumor model. Mol Imaging. 2012;11(4):286-300.

25. Schmieder AH, Caruthers SD, Zhang H, et al. Three-dimensional MR mapping of angiogenesis with alpha5beta1(alpha nu beta3)-targeted theranostic nanoparticles in the MDA-MB-435 xenograft mouse model. FASEB J. 2008;22(12):4179-4189.

26. Laurent S, Forge D, Port M, et al. Magnetic iron oxide nanoparticles: synthesis, stabilization, vectorization, physicochemical characterizations, and biological applications. Chem Rev. 2008;108(6):2064-2110.

27. Cui Y, Liu H, Liang S, et al. The feasibility of $18 \mathrm{~F}-\mathrm{AlF}-\mathrm{NOTA}-\mathrm{PRGD} 2$ $\mathrm{PET} / \mathrm{CT}$ for monitoring early response of Endostar antiangiogenic therapy in human nasopharyngeal carcinoma xenograft model compared with 18F-FDG. Oncotarget. 2016;7(19):27243-27254.

28. Sun X, Yan Y, Liu S, et al. 18F-FPPRGD2 and 18F-FDG PET of response to Abraxane Ther. 2011;52(1):140-146.

29. Bogdanowich-Knipp SJ, Chakrabarti S, Williams TD, Dillman RK, Siahaan TJ. Solution stability of linear vs. cyclic RGD peptides. $J$ Pept Res. 1999;53(5):530-541.

30. Terry SY, Abiraj K, Lok J, et al. Can 111In-RGD2 monitor response to therapy in head and neck tumor xenografts? J Nucl Med. 2014;55(11): 1849-1855.

31. Schmieder AH, Winter PM, Williams TA, et al. Molecular MR imaging of neovascular progression in the Vx2 tumor with alphavbeta3-targeted paramagnetic nanoparticles. Radiology. 2013;268(2):470-480.

32. Wen QL, Meng MB, Yang B, et al. Endostar, a recombined humanized endostatin, enhances the radioresponse for human nasopharyngeal carcinoma and human lung adenocarcinoma xenografts in mice. Cancer Sci. 2009;100(8):1510-1519.

33. Guan Y, Li A, Xiao W, et al. The efficacy and safety of Endostar combined with chemoradiotherapy for patients with advanced, locally recurrent nasopharyngeal carcinoma. Oncotarget. 2015;6(32): 33926-33934.

34. Zhou J, Wang L, Xu X, Tu Y, Qin S, Yin Y. Antitumor activity of Endostar combined with radiation against human nasopharyngeal carcinoma in mouse xenograft models. Oncol Lett. 2012;4(5):976-980.
International Journal of Nanomedicine

\section{Publish your work in this journal}

The International Journal of Nanomedicine is an international, peerreviewed journal focusing on the application of nanotechnology in diagnostics, therapeutics, and drug delivery systems throughout the biomedical field. This journal is indexed on PubMed Central, MedLine, CAS, SciSearch ${ }^{\circledR}$, Current Contents ${ }^{\circledR} /$ Clinical Medicine,

\section{Dovepress}

Journal Citation Reports/Science Edition, EMBase, Scopus and the Elsevier Bibliographic databases. The manuscript management system is completely online and includes a very quick and fair peer-review system, which is all easy to use. Visit http://www.dovepress.com/ testimonials.php to read real quotes from published authors. 